\title{
GENERALIZED ITERATION OF FORCING
}

\author{
M. GROSZEK AND T. JECH
}

\begin{abstract}
Generalized iteration extends the usual notion of iterated forcing from iterating along an ordinal to iterating along any partially ordered set. We consider a class of forcings called perfect tree forcing. The class includes Axiom A forcings with a finite splitting property, such as Cohen, Laver, Mathias, Miller, Prikry-Silver, and Sacks forcings. If $\mathscr{P}$ is a perfect tree forcing, there is a decomposition $\mathscr{Q} * \mathscr{R}$ such that $\mathscr{Q}$ is countably closed, $\mathscr{R}$ has the countable chain condition, and $\mathscr{Q} * \mathscr{R}$ adds a $\mathscr{P}$-generic set.
\end{abstract}

Theorem. The mixed-support generalized iteration of perfect tree forcing decompositions along any well-founded partial order preserves $\omega_{1}$.

Theorem. If ZFC is consistent, so is $\mathrm{ZFC}+2^{\omega}$ is arbitrarily large + whenever $\mathscr{P}$ is a perfect tree forcing and $\mathscr{D}$ is a collection of $\omega_{1}$ dense subsets of $\mathscr{P}$, there is a $\mathscr{D}$-generic filter on $\mathscr{P}$.

\section{INTRODUCTION}

Generalized iterations encompass not only iterations but also product forcings and other forcing constructions which partake of the nature of each.

Suppose $X$ is any partially ordered set. An iteration along the partial order $X$ should add to the ground model $M$ a generic sequence, $G=\langle G(x) \mid x \in X\rangle$; each $G(x)$ should be generic over $M[\langle G(y) \mid y<x\rangle]$, and if $Y$ is an initial segment of $X$ then $G \uparrow Y$ should be added by an iteration along $Y$. If $X$ is an ordinal we are talking about a standard iteration, and if the ordering on $X$ is trivial, our definition is that of a product forcing. The specific forcings of [7] and [8], designed to accomplish iterations along other partial orders, are examples of situations in which the natural forcing construction is a generalized iteration.

Generalized iteration shares in the advantages and disadvantages of both product forcing and standard iteration. For example, assuming GCH in the ground model, the countable support iteration of Sacks forcing [17] along $\omega_{2}$ preserves cardinals [2]. The same is true if $\omega_{2}$ is replaced by any well-founded partial order $X$ which is $\omega_{2}$-like (i.e., for any $x \in X$ there are at most $\omega_{1}$ points below $x$ ). Suppose that $X$ is also $\omega_{2}$-directed (i.e., any size $\omega_{1}$ subset

Received by the editors April 20, 1988.

1980 Mathematics Subject Classification (1985 Revision). Primary 03E35, 03E40, $03 E 50$.

Both authors acknowledge support from the National Science Foundation. 
of $X$ has an upper bound in $X$ ) and has size $\kappa$, where $\kappa$ is greater than $\omega_{2}$, and consider the countable support iteration of Sacks forcing along $X$. Like product forcing, it adds $\kappa$ Sacks reals without collapsing cardinals. Like iteration of length $\omega_{2}$, it produces a model in which any size $\omega_{1}$ set of reals is contained in some intermediate extension $N$, and there is a real which is Sacks generic over $N$.

This seems to hint that generalized iteration could offer an approach to obtaining consistency results with large continuum, where standard iterations have been limited to producing models in which the continuum is $\omega_{2}$. In fact, this is only a partially successful method of dealing with that general problem. The limitation is the extent to which generalized iteration encompasses product forcing. Product forcing tends to add extraneous objects to the model, evading the tight control of a standard iteration. Thus, while generalized iteration is well suited to producing models which are rich in partially generic objects, it is less easily adapted to situations in which some of this potential richness must be avoided.

The following two examples illustrate these two situations.

Forcing principles are internal forcing axioms which assert that the universe of sets is rich. They include forcing principles related to Martin's Axiom [14], generalizations of MA [18], and the proper forcing axiom [4]. The forcing principle $\operatorname{FP}(\mathscr{C}, \kappa)$ states that if $\mathscr{P}$ is any forcing partial order in the class $\mathscr{C}$, and $\mathscr{D}$ is a collection of less than $\kappa$ dense subsets of $\mathscr{P}$, then there is a $\mathscr{D}$-generic filter on $\mathscr{P}$. Baumgartner's forcing construction of a model of $\operatorname{FP}\left(\mathscr{C}, \omega_{2}\right)$, where $\mathscr{C}$ is the class of Axiom A partial orders, is an iteration whose length is a weakly compact cardinal (it is shown that the weakly compact is necessary) and produces a model in which the continuum is $\omega_{2}$. These two properties (using a large cardinal and making the continuum $\omega_{2}$ ) are common to nearly all forcings designed to produce models of forcing principles (with the outstanding exception of Martin's Axiom [14]).

A generalized iteration can produce, from a model of ZFC, a model in which the continuum is arbitrarily large and, for a certain subclass $\mathscr{B}$ of Axiom A partial orders, $\operatorname{FP}\left(\mathscr{B}, \omega_{2}\right)$ holds. The strategy, as suggested above, is to iterate forcing partial orders from $\mathscr{B}$ along an $\omega_{2}$-like, $\omega_{2}$-directed partial ordering; the technical problem is to choose $\mathscr{B}$ so that a suitable iteration will not collapse cardinals. (As the existence of incomparable points in $X$ means that the iteration will include some product forcing, $\mathscr{B}$ clearly cannot include all countable chain condition forcings, for example.) The use of generalized iteration allows us not only to make the continuum arbitrarily large but also to avoid the use of large cardinals.

To argue that the desired forcing principle holds in the generic extension, consider a relevant $\mathscr{P}$ and $\mathscr{D}$, and reflect to an initial segment of the iteration; if the next step in the iteration is to force with $\mathscr{P}$, then that adds the required $\mathscr{D}$-generic filter. In the case of a standard iteration, the next step might have been to force, not with $\mathscr{P}$, but with some other forcing partial order; a large 
cardinal reflection argument guarantees that at least one opportunity to take care of $\mathscr{P}$ and $\mathscr{D}$ was taken. In the case of a non-well-ordered iteration, there can be many "next steps"; we can force with everything in sight, and thus be assured that $\mathscr{P}$ and $\mathscr{D}$ are taken care of at the first opportunity.

An example indicating some limitations of these techniques of generalized iteration is the question of the Borel conjecture. The Borel conjecture states that there are no uncountable sets of reals with strong measure zero. Laver [13] showed this statement to be consistent, in a model with the continuum $\omega_{2}$. The problem of obtaining a model of the Borel conjecture with the continuum large was long considered (until solved by Woodin [20] with a quite specific construction) to be a paradigm of such problems.

The natural approach to attacking this question with a generalized iteration is, once again, to iterate Laver forcing along an $\omega_{2}$-like, $\omega_{2}$-directed partial order. This can be done without collapsing cardinals. In the resulting model, every set of reals of size $\omega_{1}$ is contained in some intermediate extension, over which a Laver generic real is added. This does not produce the desired result, because, as Laver comments, strong measure zero sets can be killed (as by adding a Laver real) but can also be resurrected (as by adding a Cohen real). Because the product of two copies of Laver forcing adds a Cohen real, in the generalized iteration we have just described, any size $\omega_{1}$ strong measure zero set that turns up will be continually killed off in intermediate extensions, by Laver forcing, and brought back to life in further extensions, by Cohen forcing. It is clear, from the $\Pi_{2}$ nature of the definition of strong measure zero, that such a set will have strong measure zero in the final model.

This paper is organized in four main sections, dealing with generalized iteration, perfect tree forcing, iterated perfect tree forcing, and the relative consistency of $\mathrm{FP}\left(\right.$ perfect tree forcing, $\left.\omega_{2}\right)$ with large continuum.

The definition of generalized iteration is modeled after the definition of a standard iteration as in [1]; thus if $\mathscr{P}_{X}$ is an iteration along $X$, a condition $p$ is a function with domain $X$, such that each $p(x)$ is a term for an element of the generic set $G(x)$. The details of the definition state some obviously desirable properties, having the consequences that $G(x)$ is in fact generic over $M[\langle G(y) \mid y<x\rangle]$ and that if $Y \subseteq X$ is downward-closed then the obvious restriction $\mathscr{P}_{Y}$ is an iteration along $Y$, is an initial segment of $\mathscr{P}_{X}$ (in the sense that the Boolean algebra associated with $\mathscr{P}_{Y}$ is a complete subalgebra of that associated with $\mathscr{P}_{X}$, implying a factoring lemma), and adds the generic sequence $\langle G(y) \mid y \in Y\rangle$. In $\S 1$, we give this definition and state some lemmas involving closure and chain conditions (essentially, the collection of standard lemmas that hold for both standard iterations and products). Finally, looking ahead to later constructions, we define a specific sort of "mixed-support" iteration along an $\omega_{2}$-like partial order and state some of its important properties. We make use of some ideas from Baumgartner's proof that standard countable support iteration of Axiom A forcing in length $\omega_{2}$ preserves cardinals under $\mathrm{GCH}[1]$ and from Laver's construction in [13]. 
In $\S 2$, we define the perfect tree property. Forcing partial orders with the perfect tree property include Cohen [3], Laver [13], Mathias [15], Miller [16], Prikry-Silver [6], and Sacks [17] forcings. All of these satisfy Axiom A [1] and have a finite splitting property analogous to that of Sacks forcing: to build a condition $s$ which $\leq_{n}$-extends a given condition $t$, split $t$ up into finitely many extensions (whose join is $t$ ), extend each of those individually, and then paste them back together to get $s$. Perfect tree forcing attempts to isolate this quality. In this section, we also describe a method of adding a $\mathscr{P}$-generic, where $\mathscr{P}$ is a perfect tree forcing, by first forcing with a countably closed $\mathscr{Q}$ to add a countable chain condition suborder $\mathscr{R}$ of $\mathscr{P}$, and then forcing with $\mathscr{R}$. This decomposition draws its original inspiration from Jensen's forcing construction of a definable minimal degree [10], in which $\mathscr{P}$ is Sacks forcing, and instead of forcing with $\mathscr{Q}$, he constructs $\mathscr{R}$ by using $\diamond$ to meet the relevant dense sets.

In $\S 3$, we show that the mixed-support generalized iteration of perfect tree forcing decompositions preserves $\omega_{1}$. Here, mixed support means that the countably closed $(\mathscr{Q})$ part has countable support, and the countable chain condition $(\mathscr{R})$ part has finite support. Basically, we bound a term for a countable ordinal by doing a fusion argument within the countable support part of a condition, using the finite splitting property to anticipate further finite support parts of any further extension. That any uniform method of iterating these forcings while preserving $\omega_{1}$ must rely on some such notion of mixed support could be predicted by considering methods of doing product forcing while preserving $\omega_{1}$. (For Cohen forcing, finite support works and countable support does not; for Sacks forcing, exactly the opposite; for Mathias forcing, neither works, but a mixed-support product similar to the mixed-support iteration described in [1] does.)

In the final section, we prove Theorem 15,

$\operatorname{Con}(\mathrm{ZF}) \Rightarrow \operatorname{Con}\left(\mathrm{ZFC}+\mathrm{FP}\left(\right.\right.$ perfect tree forcing, $\left.\omega_{2}\right)$

$+2^{\omega}$ is arbitrarily large),

by the methods outlined above.

We assume familiarity with the technique of iterated forcing [1] and follow, to the extent that they are appropriate, the notational conventions of [1]. In particular, $p \leq q$ means $p$ is stronger than $q$, or $p$ extends $q$. Many of the usual subtleties we obscure without remarking upon, except where they may be of genuine concern. The forcing partial orders we describe, for example, are actually preorderings; as usual, we assume that we pass to the partial order of equivalence classes by identifying conditions $p$ and $q$ if $p \leq q$ and $q \leq p$. This fact is not commented on except in the proof of Lemma 6, where we are concerned with the size of a partial order. We use no special notation to distinguish among terms for objects in various models; if we want to emphasize that the term $a$ is to be interpreted in the model $N$, we denote the corresponding object $(a)^{N}$. The rest of our notational conventions are corollaries of the policy that a given symbol should always be used to denote the same sort of object; 
thus, $s$ and $t$ refer to perfect trees, $\sigma$ and $\tau$ to elements of $\stackrel{\omega}{-2}, \mathscr{P}$ and $\mathscr{Q}$ to forcing partial orders.

\section{Generalized iteration}

If $X$ is any partial order, an iteration along $X$ should be a partial order that adds to the ground model $M$ a generic set, $\langle G(x) \mid x \in X\rangle$, with the property that each $G(x)$ is generic over $M[\langle G(y) \mid y<x\rangle]$ for some partial order $\mathscr{Q}_{x}$. In case $X$ is an ordinal, our definition will reduce to that of a standard iteration; if the ordering on $X$ is trivial, a product forcing.

The definition below is modeled after the definition of a standard iteration (as in, e.g., [1]). Thus, a condition is a function $p$ with domain $X$, each $p(x)$ being a term for a condition in $\mathscr{Q}_{x}$; a stronger condition $q$ (which we denote $q \leq p)$ extends $p$ on each coordinate.

We refer to partial orders along which we iterate as support partial orders, and partial orders with which we force as forcing partial orders.

Definition. Let $X$ be any support partial order, and $\mathscr{P}$ a forcing partial order whose elements are functions with domain $X$. For $Y$ a downward-closed subset of $X, x \in X$, and $p \in \mathscr{P}$, let

$$
\begin{gathered}
\mathscr{P}_{Y}=\{p \uparrow Y \mid p \in \mathscr{P}\}, \\
X \uparrow x=\{y \mid y<x\} \text { and } X(x)=\{y \mid y \leq x\}, \\
\mathscr{P} \uparrow x=\mathscr{P}_{X \uparrow x} \text { and } \mathscr{P}(x)=\mathscr{P}_{X(x)}, \\
p \uparrow x=p \uparrow(X \uparrow x) .
\end{gathered}
$$

Then $\mathscr{P}$ is an iteration along $X$ provided:

(i) If $Y \subseteq X$ is downward-closed, and $\mathscr{P}_{Y}$ is given the induced ordering

$$
\begin{gathered}
p \leq q \text { iff there are } \bar{p} \text { and } \bar{q} \text { in } \mathscr{P}_{X} \text { such that } \\
\bar{p} \uparrow Y=p, \quad \bar{q} \uparrow Y=q, \quad \text { and } \quad \bar{p} \leq \bar{q},
\end{gathered}
$$

then whenever $p \in \mathscr{P}, q \in \mathscr{P}_{Y}, q \leq p \uparrow Y$, and we define

then $\bar{p} \in \mathscr{P}$.

$$
\bar{p}(y)= \begin{cases}q(y), & y \in Y, \\ p(y), & y \notin Y,\end{cases}
$$

Whenever $p \in \mathscr{P}, q \in \mathscr{P}$, and $p \nmid X-Y=q \uparrow X-Y$, then $p \leq q$ iff $p \uparrow$ $Y \leq q \uparrow Y$.

(ii) There is an indexed set of terms $\left\langle\mathscr{Q}_{x} \mid x \in X\right\rangle$ such that for each $x \in X$, $\mathscr{P}(x)$ is the two-step iteration $\mathscr{P} \uparrow x * \mathscr{Q}_{x}$.

(iii) The maximal element of $\mathscr{P}$ is the condition 1 , where for every $x \in X$, $1(x)$ is forced to be the maximal element of $\mathscr{Q}_{x}$ (also denoted 1 ).

This implies that for downward-closed $Y \subseteq X, 1 \uparrow Y$ is the maximal element of $\mathscr{P}_{Y}$.

(iv) For any $p$ and $q$ in $\mathscr{P}, p \leq q$ iff for every $x \in X, p \uparrow x \leq q \uparrow x$ and $p \nmid x \Vdash p(x) \leq q(x)$. 
This definition implies that if $Y \subseteq X$ is downward-closed, then $\mathscr{P}_{Y}$ is an iteration along $Y . \mathscr{P}_{X}$ will always denote a forcing partial order which is an iteration along the support partial order $X$.

Forcing with $\mathscr{P} \uparrow x$ may be denoted $\Vdash_{x}$.

If $\mathscr{G} \subseteq \mathscr{P}_{X}$ is generic over the ground model $M$, and $Y \subseteq X$ is downwardclosed, then

is generic for $\mathscr{P}_{Y}$ over $M$. We may denote

$$
\mathscr{G}_{Y}=\{p|Y| p \in \mathscr{G}\}
$$

$$
M_{Y}=M\left[\mathscr{G}_{Y}\right]
$$

The set $\mathscr{G}$ is equivalent to $G=\langle G(x) \mid x \in X\rangle$, sometimes called the generic sequence, defined by

$$
G(x)=\left\{(p(x))^{M_{X \mid x}} \mid p \in \mathscr{G}\right\} .
$$

We also denote, for $Y \subseteq X$ and $x \in X$,

$$
G_{Y}=G \nmid Y \text { and } G \nmid x=G \uparrow(X \nmid x) .
$$

If $X$ is an ordinal $\alpha$, then an iteration along $X$ is an iteration in the usual sense. If all the points of $X$ are incomparable, then an iteration along $X$ is a product of the forcing partial orders $\left\langle\mathscr{Q}_{x} \mid x \in X\right\rangle$.

This definition does not have the inductive nature of the definition of a standard iteration, allowing for the possibility that $X$ is ill-founded. Although an ill-founded iteration is appropriate in some contexts (Easton forcing [5] can be regarded as one, as can the forcing partial order of [7]), in general, merely producing an iteration along an ill-founded partial order poses a challenge which we are content to avoid.

Definition. From this point on, all support partial orders are well founded.

Even for a well-founded support partial order $X, \mathscr{P}_{X}$ generally cannot be viewed as a standard iteration, the $\alpha$ th component of which is some product of the $\left\langle\mathscr{Q}_{x}\right|$ height $\left.(x)=\alpha\right\rangle$. This is because the term $p(x)$ can only depend on $G \backslash x$, an important point when dealing with generalized iteration.

As with standard iteration, however, we can now define $\mathscr{P}_{X}$ inductively and apply inductive proofs. Similarly, we can specify $\mathscr{P}_{X}$ by giving $\left\langle\mathscr{Q}_{x} \mid x \in X\right\rangle$ and the type of supports to be required.

Definition. For $p \in \mathscr{P}_{X}$, the support of $p$ is

$$
\operatorname{supp}(p)=\{x \mid \neg(p \mid x \Vdash p(x)=1)\} .
$$

Definition. Suppose $\mathscr{P}_{X}$ is an iteration along $x$ of $\left\langle\mathscr{Q}_{x} \mid x \in X\right\rangle$, and $\mathscr{Y}$ is a collection of subsets of $X$, such that $\mathscr{Y}$ contains all finite subsets of $X, \mathscr{Y}$ is closed under taking subsets and under unions (i.e., $\mathscr{Y}$ is an ideal in the power set of $X, \mathscr{P}(X))$. Then $\mathscr{P}_{X}$ is the iteration of $\left\langle\mathscr{Q}_{x} \mid x \in X\right\rangle$ with supports from $\mathscr{Y}$ iff

$$
\begin{aligned}
& \mathscr{P}_{X}=\{p \mid p \text { is a function with domain } X, \operatorname{supp}(p) \in \mathscr{Y}, \\
& \text { and for all } \left.x \in X, p|X(x) \in \mathscr{P}| x * \mathscr{Q}_{x}\right\} .
\end{aligned}
$$


Instead of the above, we could have chosen to follow the strategy of a standard iteration, by defining $\mathscr{P}_{X}$ from $\mathscr{Y}$ and $\left\langle\mathscr{Q}_{x} \mid x \in X\right\rangle$, by induction on the height of $X$. One way to do this is to set

$$
\begin{aligned}
& \mathscr{P}_{X}=\{p \mid p \text { is a function with domain } X, \\
& \qquad \forall x \in X)[p \nmid x \text { is in the }\{Y \cap X|x| Y \in \mathscr{Y}\} \\
& \text { support iteration of }\left\langle\mathscr{Q}_{y} \mid y<x\right\rangle, \\
& \left.\left.\quad \text { and } p \nmid x \Vdash p(x) \in \mathscr{Q}_{x}\right], \text { and } \operatorname{supp}(p) \in \mathscr{Y}\right\} .
\end{aligned}
$$

As usual, we may refer to $\kappa$-support or less-than- $\kappa$-support iterations where $\kappa$ is any cardinal.

Since generalized iteration encompasses product forcing, it does not preserve cardinals as easily as standard iteration. The following lemmas, however, involving closure and cardinality arguments, can be lifted directly from the corresponding proofs for standard iterations and products.

Definition. Let $X$ be a partial order and $\kappa$ a cardinal. $X$ is $\kappa$-like iff for every $x \in X, \operatorname{CARD}(X \mid x)<\kappa$.

For the rest of this section, let $X$ denote a support partial order, $\mathscr{Y}$ an ideal in $\mathscr{P}(X), \kappa$ and $\lambda$ regular cardinals.

Lemma 1. If $\mathscr{Z}$ is a collection of downward-closed subsets of $X ; \mathscr{Z}$ is closed under unions of size less that $\kappa ;$ for every $Z$ in $\mathscr{Z}, \mathscr{P}_{Z}$ is $\kappa$-closed; and $\mathscr{P}_{X}$ is the union of $\left\{\mathscr{P}_{Z} \mid Z \in \mathscr{Z}\right\}$; then $\mathscr{P}_{X}$ is $\kappa$-closed.

Lemma 2. If $\mathscr{Z}$ is a collection of downward-closed subsets of $X$; for every $Z$ in $\mathscr{Z}, \mathscr{P}_{Z}$ is $\kappa$-closed ; and

$\mathscr{P}_{X}=\left\{p \mid p\right.$ is a function with domain $X$, and $\left.(\forall Z \in \mathscr{Z})\left[p \nmid Z \in \mathscr{P}_{Z}\right]\right\}$;

then $\mathscr{P}_{X}$ is $\kappa$-closed.

Lemma 3. If $\mathscr{P}_{X}$ is an iteration with supports from $\mathscr{Y} ; \mathscr{Y}$ is closed under unions of size less than $\kappa$; and each $\mathscr{Q}_{x}$ is forced to be $\kappa$-closed; then $\mathscr{P}_{X}$ is $\kappa$-closed.

(This lemma is proved by induction on the height of $X$. Let $Z(\beta)=$ $\{x \mid \operatorname{ht}(x)<\beta\}$. If $\operatorname{ht}(X)$ is a limit ordinal, apply Lemma 1 or Lemma 2 , depending on the cofinality of $\operatorname{ht}(X)$, with $\mathscr{Z}=\{Z(\beta) \mid \beta<\operatorname{ht}(X)\}$. If $\operatorname{ht}(X)=\alpha+1$, use the fact that $\mathscr{P}_{Z(\alpha)}$ and each $\mathscr{Q}_{x}$ for $x$ of height $\alpha$ are $\kappa$-closed.)

Lemma 4. Assume $G C H, \kappa$ and $\lambda$ are regular cardinals. If $\kappa<\lambda, X$ is $\lambda$-like, $\mathscr{P}_{X}$ is an iteration along $X$ for which all supports have size less than $\kappa$, and whenever $Y \subset X$ has cardinality less than $\kappa, \operatorname{CARD}\left(\mathscr{P}_{Y}\right)<\lambda$, then $\mathscr{P}_{X}$ has the $\lambda$-chain condition.

(The above lemma is proved using a standard delta system argument.) 
Now we are about to define a kind of mixed-support iteration. The intention is for each forcing partial order $\mathscr{Q}_{x}$ to add a generic subset of some standard forcing $\mathscr{P}$ to add a real; $\mathscr{Q}_{x}$ will not be $\mathscr{P}$ itself, but an associated forcing partial order. The next section will be devoted to giving a property of $\mathscr{P}$ which will guarantee that $\omega_{1}$ is preserved by the iteration.

Definition. Let $X$ be any support partial order, and $\mathscr{P}_{X}$ an iteration along $X$.

Suppose that for each $x \in X$, the forcing partial order at coordinate $x$ is a two-step iteration. Instead of $\mathscr{Q}_{x}$, denote it by $\mathscr{Q}_{x} * \mathscr{R}_{x}$. For $p \in \mathscr{P}_{X}$, since $p(x)$ is forced to be in $\mathscr{Q}_{x} * \mathscr{R}_{x}$, we can write

$$
p(x)=\left\langle p^{Q}(x), p^{R}(x)\right\rangle
$$

and define the root of $p$ to be

$$
\operatorname{root}(p)=\left\{x \mid \neg\left(p \nmid x \Vdash\left(p^{Q}(x) \Vdash p^{R}(x)=1\right)\right)\right\} .
$$

Then, inductively on the height of $X$, define the mixed-support iteration of $\left\langle\mathscr{Q}_{x} * \mathscr{R}_{x} \mid x \in X\right\rangle$ to be the iteration $\mathscr{P}_{X}$ such that

(i) For all $x \in X, \mathscr{P} \uparrow x$ is the mixed-support iteration of $\left\langle\mathscr{Q}_{y} * \mathscr{R}_{y}\right| y\langle x\rangle$, and $\mathscr{P} \uparrow X(x)$ is $\mathscr{P} \uparrow x * \mathscr{Q}_{x} * \mathscr{R}_{x}$.

(ii) $\mathscr{P}_{X}=\left\{p \mid p\right.$ is a function on $X$, for all $x \in X, p\left\lceil X(x) \in \mathscr{P}_{X(x)}\right.$, $\operatorname{supp}(p)$ is countable, and root $(p)$ is finite $\}$.

Definition. If $\mathscr{P}_{X}$ is a mixed-support iteration, and $p$ and $q$ are in $\mathscr{P}_{X}$, define " $p$ "-extends $q$ " by

$$
p \leq^{*} q \text { iff } p \leq q \text { and for all } x \in X, p \nmid x \Vdash p^{R}(x)=q^{R}(x) .
$$

Lemma 5. If $\mathscr{P}_{X}$ is a mixed-support iteration for which all $\mathscr{Q}_{x}$ are forced to be countably closed, and $\left\langle p_{n}\right| n\langle\omega\rangle$ is a sequence from $\mathscr{P}_{X}$ such that for all $n$, $p_{n+1} \leq^{*} p_{n}$, then $\left\langle p_{n} \mid n<\omega\right\rangle$ has a lower bound in $\mathscr{P}_{X}$.

Definition. $D \subset \mathscr{P}_{X}$ is said to be ${ }^{*}$-dense in $\mathscr{P}_{X}$ iff for every $p \in \mathscr{P}_{X}$ there is a $q \in D$ such that $q \leq^{*} p$.

Lemma 6. Suppose $\mathscr{P}_{X}$ is a mixed-support iteration, all $\mathscr{Q}_{x}$ are forced to be countably closed, and for any antichain $A \subset \mathscr{P}_{X}$

$$
\{p \mid\{q \in A \mid q \text { and } p \text { are compatible }\} \text { is countable }\}
$$

is ${ }^{*}$-dense in $\mathscr{P}_{X}$. (Equivalently, we could say that for any term a forced to lie in the ground model,

$\{p \mid$ there is a countable ground model set $B$ such that $p \Vdash a \in B\}$

is ${ }^{*}$-dense in $\mathscr{P}_{X}$ ). Then $\mathscr{P}_{X}$ preserves $\omega_{1}$.

Suppose further that each $\mathscr{Q}_{x} * \mathscr{R}_{x}$ is forced to have size $\omega_{1}$ and that in the ground model, GCH holds. Then: 
If $\operatorname{CARD}(X) \leq \omega_{1}$, then $\mathscr{P}_{X}$ has a ${ }^{*}$-dense subset of size $\omega_{1}$, and $\mathscr{P}_{X}$ preserves cardinals and $G C H$.

If $X$ is $\omega_{2}$-like, then $\mathscr{P}_{X}$ has the $\omega_{2}$-chain condition, and $\mathscr{P}_{X}$ preserves cardinals.

Proof. The only part of this lemma which does not immediately follow from preceding lemmas and standard facts about forcing is the claim that under the cited hypothesis $\mathscr{P}_{X}$ has a ${ }^{*}$-dense subset $\overline{\mathscr{P}}_{X}$ of size $\omega_{1}$. This is like the analogous fact for Axiom A forcing. Inductively on the height of $X$ we define $\overline{\mathscr{P}}_{X}$ :

For all $x \in X$, let $f_{x}$ be a term for an enumeration of $\mathscr{Q}_{x} * \mathscr{R}_{x}$ in type $\omega_{1}$. Then let

$$
\begin{aligned}
\mathscr{P}_{X}=\{p \mid & \text { for all } x, \text { the value of } f_{x}^{-1}(p(x)) \\
& \text { is decided by a countable antichain in } \overline{\mathscr{P}}\lceil x\} .
\end{aligned}
$$

Then, assuming we identify conditions $p$ and $q$ if $p \leq q$ and $q \leq p$, it is not hard to show inductively that $\overline{\mathscr{P}}_{X}$ is indeed a *-dense subset of $\mathscr{P}_{X}$ of size $\omega_{1}$.

\section{Perfect tree Forcing}

We now want to describe a property of $\mathscr{Q}_{x} * \mathscr{R}_{x}$ which will guarantee that $\mathscr{P}_{X}$, the mixed-support iteration of $\left\langle\mathscr{Q}_{x} * \mathscr{R}_{x} \mid x \in X\right\rangle$, preserves $\omega_{1}$. We will design $\mathscr{Q}_{x} * \mathscr{R}_{x}$ corresponding to a given forcing partial order $\mathscr{P}$, to add a generic subset of $\mathscr{P}$. ( $\mathscr{P}$ can be any one of a certain class of Axiom A partial orders, among them the forcings to add reals introduced by Cohen, Laver, Mathias, Miller, Prikry and Silver, and Sacks.) The countably closed $\mathscr{Q}_{x}$ will generically add $\mathscr{R}_{x} \subset \mathscr{P}$ with the property that any dense open subset of $\mathscr{P}$ intersects $\mathscr{R}_{x}$ in a dense set. In addition, $\mathscr{Q}_{x}$ will take advantage of the fusion arguments that prove $\mathscr{P}$ preserves $\omega_{1}$, and will impose enough control on the fusion process to prove that the generalized iteration preserves $\omega_{1}$.

Generalized iteration of each of the individual forcings mentioned above can be treated in a simpler manner than that described below: Cohen forcing by using finite supports, Sacks forcing by using countable supports, Mathias forcing by using a different notion of mixed support. The point of the following definition is to provide a uniform method of iterating a class of forcings including all of these, both for its own sake and to allow iterations that combine different such forcings, as in the proof of Theorem 15.

Definition. If $t \subset{ }^{\omega} 2$ and $\sigma \in t$, we say that $\sigma$ splits in $t$ iff both $\sigma^{\curvearrowright} 0$ and $\sigma^{\frown} 1$ are in $t$.

A perfect tree is a subset $t$ of ${ }^{\omega} 2$ which is downward-closed and such that whenever $\sigma \in t$ there is an extension of $\sigma$ which splits in $t$.

If $t$ is a perfect tree, the stem of $t, \operatorname{stem}(t)$, is the least $\sigma$ which splits in $t$. The $n$th splitting level of $t$ is

$$
S_{n}(t)=\{\sigma \mid \sigma \text { splits in } \tau \text { and } \operatorname{CARD}(\{\tau \subsetneq \sigma \mid \tau \text { splits in } t\})=n\} .
$$


Hence $\operatorname{stem}(t)$ is the unique element of $S_{0}(t)$.

If $t$ is a perfect tree and $\sigma \in t$, the restriction of $t$ to $\sigma$ is

$$
(t)_{\sigma}=\{\tau \in t \mid \tau \subseteq \sigma \text { or } \sigma \subset \tau\} .
$$

Let $\mathscr{T}$ be the set of all perfect trees, ordered by

$$
s \leq t \quad \text { iff } \quad s \subseteq t .
$$

Definition. A forcing partial order $\mathscr{P}$ has the perfect tree property provided:

(i) $\mathscr{P}$ is a suborder of $\mathscr{T}$ which is closed under restrictions and under changes of stem (i.e., if $s \in \mathscr{P}, \sigma, \tau \in{ }^{n} 2$, and $\sigma=\operatorname{stem}(s)$, the tree obtained by changing the stem of $s$ to $\tau$,

$$
\left\{\tau\left\ulcorner p \mid \sigma^{\frown} p \in s\right\}\right.
$$

is also in $\mathscr{P})$.

(ii) (Zero-extensions) There is a refinement of $\leq, \leq_{0}$, such that:

whenever $s \leq t, \tau \in s$, and $\operatorname{stem}(s) \subseteq \tau$, then $(s)_{\tau} \leq_{0}(t)_{\tau}$;

whenever $s \leq_{0} t$, and $\bar{s}$ and $\bar{t}$ are obtained from $s$ and $t$ by replacing $\operatorname{stem}(s)$ with $\sigma$, then $\bar{s} \leq_{0} \bar{t}$.

(In most applications, $s \leq_{0} t$ intuitively means that $s \leq t$ and $s$ and $t$ have the same finite part, possibly the same stem.)

This induces further refinements,

$$
s \leq_{n} t \quad \text { iff } \quad s \leq_{0} t \& S_{n}(t) \subset s .
$$

If $S \leq_{n} t$, we say that $s n$-extends $t$. As a consequence of this definition,

$$
s \leq_{n} t \Rightarrow \text { for all } \sigma \in S_{n}(t),(s)_{\sigma} \leq_{0}(t)_{\sigma} .
$$

(iii) (Amalgamations) Suppose $s$ and $t$ are elements of $\mathscr{P}, \sigma \in S_{n}(t)$, and $s \leq_{0}(t)_{\sigma}$. Then there is a condition $r$, called the amalgamation of $s$ into $t$ and denoted $\operatorname{Am}_{n}(s, t)$, such that:

$$
r \leq_{n} t \text { and }(r)_{\sigma} \leq_{0} s
$$

for all $\tau \in S_{n}(t),(r)_{\tau}$ is obtained either from $s$ or from $(t)_{\tau}$ for some $\tau$ by a change of stem.

(iv) (Fusions) Suppose $\left\langle t_{n} \mid n<\omega\right\rangle$ is a sequence of conditions in $\mathscr{P}$ such that for any $n, t_{n+1} \leq_{n} t_{n}$. Then $t_{\omega}=\bigcap\left\{t_{n} \mid n<\omega\right\}$ is in $\mathscr{P}$. Such a sequence is called a fusion sequence, and $t_{\omega}$ is called its fusion.

Lemma 7. If $\mathscr{P}$ has the perfect tree property, then $\mathscr{P}$ preserves $\omega_{1}$.

Proof. In fact, if $\mathscr{P}$ is a perfect tree forcing, then $\mathscr{P}$ satisfies Axiom A [1]. Given condition (iv) of the definition of perfect tree forcing, it is only necessary to show:

Whenever $t \in \mathscr{P}, n<\omega$, and $A$ is a maximal antichain in $\mathscr{P}$, there are a condition $s \leq_{n} t$ and a countable $B \subset A$ such that $B$ is predense in $\mathscr{P}$ below $s$. 
Suppose then that $t$ and $A$ are given. Define a fusion sequence $\left\langle t_{m} \mid m<\omega\right\rangle$ inductively on $m$.

If $m \leq n$, let $t_{m}=t$.

Suppose $t_{m}$ is given, $m \geq n$; let $S_{m}\left(t_{m}\right)=\left\{\tau(i) \mid i<2^{m}\right\}$. Inductively on $i \leq 2^{m}$ define $s(i)$ :

Let $s(0)=t_{m}$.

Given $s(i)$, if there is a condition $r \leq_{0}(s(i))_{\tau(i)}$ such that $r$ extends some element of $A$, let $s_{i+1}=\operatorname{Am}_{n}(r(s(i)))$. Otherwise, let $s(i+1)=s(i)$.

Finally, let $t_{m+1}=s\left(2^{m}\right)$. By construction, if $\sigma \in S_{m}\left(t_{m+1}\right)$ and any zeroextension of $\left(t_{m+1}\right)_{\sigma}$ extends an element of $A$, then $\left(t_{m+1}\right)_{\sigma}$ already does so. (We say we have successively zero-extended the $\left(t_{m}\right)_{\sigma}, \sigma \in S_{m}\left(t_{m}\right)$, and then amalgamated them back in; a technique that will often be used.)

Let $s$ be the fusion of the sequence $\left\langle t_{m}\right| m\langle\omega\rangle$, and

$$
B=\left\{r \in A \mid \text { for some } \sigma,(s)_{\sigma} \leq r\right\} \text {. }
$$

Then $s \leq_{n} t$, and $B$ is predense below $s$, for, suppose, $r \leq s$. By extending $r$, we can assume that $r$ extends an element of $A$, and $\operatorname{stem}(r) \in S_{n}(s)$ for some $n>m$. But then $r \leq_{0}(s)_{\text {stem }(r)}$, and so by construction $(s)_{\text {stem }(r)}$ extends an element of $B$.

This proves the lemma.

We have claimed that Cohen, Laver, Mathias, Miller, Prikry-Silver, and Sacks forcing can all be given the structure of perfect tree forcing. In the Appendix, we give details for some illustrative examples.

Now we describe the decomposition of a perfect tree forcing partial order $\mathscr{P}$ into a two-step iteration $\mathscr{Q} * \mathscr{R}$.

Roughly speaking, a condition in $\mathscr{Q}$ will be a pair $\langle P, \mathscr{A}\rangle$ such that $P$ is a countable suborder of $\mathscr{P}$ and $\mathscr{A}$ is a countable collection of predense subsets of $P$; to extend the condition, increase both coordinates. (Thus $\mathscr{Q}$ will be countably closed.) We will define $\mathscr{R}$ to be the union of all first coordinates of conditions in the $\mathscr{Q}$-generic set; thus $\langle P, \mathscr{A}\rangle$ forces " $P \subseteq \mathscr{R}$ and every element of $\mathscr{A}$ is predense in $\mathscr{R} "$. In order to carry out fusion arguments within the countable set $P$, which will be necessary to show that $\mathscr{Q}$ forces $\mathscr{R}$ to add a $\mathscr{P}$-generic set over the ground model, further restrictions must be imposed on $P$ and $\mathscr{A}$.

Definition. $\langle P, \mathscr{A}\rangle$ is a condition in $\mathscr{Q}$ provided:

(i) $P$ is a countable suborder of $\mathscr{P}$, closed under restrictions, changes of stem, and finite unions of conditions with incompatible stems (when such unions are conditions in $\mathscr{P}$ ).

(ii) $\mathscr{A}$ is a countable collection of subsets of $P$, such that whenever $A \in \mathscr{A}$ and $t \in P$, then for some $s \in P, s \leq_{0} t$ and $A$ is predense below $s$ in the larger forcing partial order $\mathscr{P}$. (For an alternative definition of $\mathscr{Q}$, one can make the stronger, and more concrete, requirement that for some $n$ and every $\sigma \in S_{n}(x),(s)_{\sigma}$ extends an element of $A$.) 


$$
\langle P, \mathscr{A}\rangle \leq\langle Q, \mathscr{B}\rangle \quad \text { iff } \quad Q \subseteq P \text { and } \mathscr{B} \subseteq \mathscr{A}
$$

Under this definition, $P$ is closed under amalgamations. It is also easy to see that $\mathscr{Q}$ is countably closed.

The next definition is that of a fusion process for $\mathscr{Q}$. We want to take a condition $\langle P, \mathscr{A}\rangle$ and extend it to $\langle Q, \mathscr{B}\rangle$, such that there is a $B \in \mathscr{B}$, all of whose elements have been constructed to have some desired property by a fusion argument. That is, while constructing a decreasing sequence of conditions $\left\langle\left\langle P_{n}, \mathscr{A}_{n}\right\rangle \mid n<\omega\right\rangle$, we simultaneously construct a collection of fusion sequences from $P_{\omega}$, in such a way that if $B$ is the set of all fusions of those sequences, there is a $\langle Q, \mathscr{B}\rangle \leq\left\langle P_{\omega}, \mathscr{A}_{\omega}\right\rangle$ with $B \in \mathscr{B}$. This definition allows some degree of freedom in certain steps of the construction (the choice of $\left\langle P_{n}, \mathscr{A}_{n}\right\rangle$ and the extension step in the construction of the fusion sequences) that will depend on what we want the predense set $B$ to accomplish.

Definition. A fusion process for $\mathscr{Q}$ is an $(\omega+1)$-stage procedure, as follows.

During the process, conditions from $\mathscr{Q},\left\langle P_{n}, \mathscr{A}_{n}\right\rangle$ for $n<\omega$, will be selected. Let $\left\{t_{n} \mid n<\omega\right\}$ and $\left\{A_{n} \mid n<\omega\right\}$ be standard diagonal enumerations of $P_{\omega}=\bigcup\left\{P_{n} \mid n<\omega\right\}$ and $\mathscr{A}_{\omega}=\bigcup\left\{\mathscr{A}_{n} \mid n<\omega\right\}$, respectively.

Begin by choosing a condition $\left\langle P_{0}, \mathscr{A}_{0}\right\rangle$.

Stage $n$. First, begin constructing a fusion sequence $\left\langle t_{n}(m) \mid m<\omega\right\rangle$ for $t_{n}$ by setting, for all $m \leq n, t_{n}(m)=t_{n}$.

Second, for each $m \leq n$, define $s_{m}(n) \leq_{n} t_{m}(n)$ so that for any $\sigma \in$ $S_{n}\left(t_{m}(n)\right)$, and any $r$ obtained from $\left(s_{m}(n)\right)_{\sigma}$ by changing $\sigma \uparrow n$, for all $j \leq n, A_{j}$ is predense below $r$ in $\mathscr{P}$. (We do this, as before, by successively zero-extending all $\left(t_{m}(n)\right)_{\sigma}$ and amalgamating them back in. $P_{n}$ has closure properties that allow us to carry out this process in $P_{n}$, and choose $s_{m}(n) \in P_{n}$.)

Third, for each $m \leq n$, choose $t_{m}(n+1) \leq_{n} s_{m}(n)$. (This is the extension step.)

Finally, choose $\left\langle P_{n+1}, \mathscr{A}_{n+1}\right\rangle \leq\left\langle P_{n}, \mathscr{A}_{n}\right\rangle$. This completes stage $n$.

Stage $\omega$. For each $m<\omega$, let $s_{m}$ be the fusion of the fusion sequence for $t_{m},\left\langle t_{m}(n) \mid n<\omega\right\rangle$ (so, in particular, $\left.s_{m} \leq_{0} t_{m}\right)$. Let

$B=\left\{s \mid s\right.$ is obtained from a restriction of some $s_{m}$ by change of stem $\}$.

Let $Q$ be the closure of $P_{\omega} \cup B$ under finite unions of conditions with incompatible stems, and let $\mathscr{B}=\mathscr{A}_{\omega} \cup\{B\}$. This completes stage $\omega$.

Claim. $\langle Q, \mathscr{B}\rangle$ is a condition in $\mathscr{Q}$.

Proof of claim. It is not hard to see that $Q$ is closed under restrictions and changes of stem. As every $A_{n}$ is, by construction, predense in $\mathscr{P}$ below every element of $B$, it suffices to show that:

For every $t \in Q$ there is a $s \in Q$ such that $s \leq_{0} t$, and $B$ is predense in $\mathscr{P}$ below $s$. 
Suppose, then, that $t \in Q$. By the expression of $t$ as a finite union, there is some $n$ such that for every $\sigma \in S_{n}(t)$, either $(t)_{\sigma} \in P_{\omega}$ or $(t)_{\sigma} \in B$. Let $S_{n}(t)=\{\tau(i) \mid i<k\}$, and inductively on $i \leq k$ define $t(i)$.

Let $t(0)=t$.

Given $t(i)$, by construction so far, either $(t(i))_{\tau(i)} \in B$ or $(t(i))_{\tau(i)} \in P_{\omega}$. If $(t(i))_{\tau(i)} \in B$, let $t(i+1)=t(i)$. Otherwise, suppose $(t(i))_{\tau(i)}=t_{m}$. Then $s_{m} \leq_{0} t_{m}$ and $s_{m} \in B$; let $t(i+1)=\operatorname{Am}_{n}\left(s_{m}, t(i)\right)$.

Finally, let $s=t(k)$. For all $\sigma \in S_{n}(s),(s)_{\sigma}$ extends an element of $B$, so $B$ is predense in $\mathscr{P}$ below $s$; and $s \leq_{0} t$. This proves the claim.

Claim. Suppose $s \in B$, and $r$ is any extension of $s$ in $\mathscr{P}$. Let

$\bar{Q}=\{t \mid t \in Q$ or $t$ is obtained from a restriction of $r$ by a change of stem $\}$, and let $R$ be the closure of $\bar{Q}$ under the usual finite unions. Then $\langle R, \mathscr{B}\rangle$ is a condition in $\mathscr{Q}$.

Proof of claim. Exactly as above. $R$ has the desired closure properties, and for any $t \in R$ there is an $n$ such that for any $\sigma \in S_{n}(t),(t)_{\sigma}$ is either in $P_{\omega}$, in $B$, or obtained from a restriction of $r$ by a change of stem (and hence an extension of an element of $B$ ).

Lemma 8. If $\mathscr{G} \subseteq \mathscr{Q}$ is generic over $M, \mathscr{R}=\bigcup\{P \mid\langle P, \varnothing\rangle \in \mathscr{G}\}$, and $D$ is a dense subset of $\mathscr{P}$ in $M$, then $D \cap \mathscr{R}$ is a dense subset of $\mathscr{R}$.

Proof. Let $\langle P, \mathscr{A}\rangle \in \mathscr{Q}$ and $t \in P$. Find $\langle R, \mathscr{B}\rangle \leq\langle P, \mathscr{A}\rangle$ such that there is $r \leq t$ in $R \cap D$ : Perform a fusion process for $\mathscr{Q}$ in which, for all $n$, $\left\langle P_{n}, \mathscr{A}_{n}\right\rangle=\langle P, \mathscr{A}\rangle$, and the extension step is to set $t_{m}(n+1)=s_{m}(n)$, (i.e., do nothing). If $t=t_{m}$, choose $r \leq s_{m}$ from $D$, and define $\langle R, \mathscr{B}\rangle$ as in the above claim.

Lemma 9. $\mathscr{R}$ as in Lemma 8 has the countable chain condition.

Proof. Let $C$ be a term for a maximal antichain in $\mathscr{R}$, and $\langle P, \mathscr{A}\rangle$ be a condition in $\mathscr{Q}$. As $\mathscr{Q}$ is countably closed, we can assume that for every $t \in P$, either there are $s \in P$ and $r \in P$ such that

$$
s \leq_{0} t, \quad s \leq r, \text { and }\langle P, \mathscr{A}\rangle \Vdash r \in C,
$$

or

$$
\langle P, \mathscr{A}\rangle \Vdash \text { "no zero-extension of } t \text { extends an element of } C \text { ". }
$$

Perform a fusion process for $\mathscr{Q}$, where for all $n\left\langle P_{n}, \mathscr{A}_{n}\right\rangle=\langle P, \mathscr{A}\rangle$, and the extension step is to find $t_{m}(n+1) \leq_{n} S_{m}(n)$ such that for every $\sigma \in$ $S_{n}\left(t_{m}(n+1)\right)$, either

$\langle P, \mathscr{A}\rangle \Vdash$ "no zero-extension of $\left(t_{m}(n+1)\right)_{\sigma}$ extends an element of $C$ ", or $\left(t_{m}(n+1)\right)_{\sigma}$ extends some $r \in P$ such that

$$
\langle P, \mathscr{A}\rangle \Vdash r \in C ;
$$


and, furthermore, the same is true of any condition obtained from $\left(t_{m}(n+1)\right)_{\sigma}$ by changing $\sigma \uparrow n$. (This can be done as usual, by looking one by one at restrictions of the condition in question to nodes on the $n$th splitting level, zero-extending them to have the desired property, and then amalgamating them back in.)

But now

$$
\langle Q, \mathscr{B}\rangle \Vdash \text { “ }\{r \mid\langle P, \mathscr{A}\rangle \Vdash r \in C \& r \in P\}=C ",
$$

which forces $C$ to be countable. To see this, let $\mathscr{G} \subset \mathscr{Q}$ be generic, $\langle Q, \mathscr{B}\rangle \in$ $\mathscr{G}$, and $\bar{r} \in C$, and show

$$
\bar{r} \in P \text { and }\langle P, \mathscr{A}\rangle \Vdash \bar{r} \in c .
$$

There is a condition $t \leq \bar{r}$ in $\mathscr{R}$ which extends some $s \in B$. Then for sufficiently large $\sigma \in t,(t)_{\sigma} \leq_{0}(s)_{\sigma}$, and, by construction, if any zero-extension of $(s)_{\sigma}$ extends an element of $C$, then for some $\overline{\bar{r}} \in P$,

$$
(s)_{\sigma} \leq_{0} \overline{\bar{r}} \text { and }\langle P, \mathscr{A}\rangle \Vdash \overline{\bar{r}} \in C .
$$

But then $(t)_{\sigma} \leq \overline{\bar{r}}$ and $(t)_{\sigma} \leq \bar{r}$; as $C$ is an antichain, $\bar{r}=\overline{\bar{r}}$, completing the proof.

\section{ITERATED PERFECT TREE FORCING}

In this section, we use the notion of a "fusion process for $\mathscr{Q}$ " (i.e., for each $\mathscr{Q}_{x}$ ), as it was defined in $\S 2$, to prove that if each $\mathscr{Q}_{x} * \mathscr{R}_{x}$ is the decomposition of a perfect tree forcing partial order, then the mixed-support iteration of $\left\langle\mathscr{Q}_{x} * \mathscr{R}_{x} \mid x \in X\right\rangle$ preserves $\omega_{1}$.

In light of $\S 1$, we need only prove that if $\mathscr{P}_{X}$ is this mixed-support iteration and $a$ is any term for an element of the ground model, then the set of conditions that force $a$ into a countable ground model set is ${ }^{*}$-dense. We will exploit the fact that the fusion process for $\mathscr{Q}$ allows us to bound antichains in $\mathscr{R}$ by considering only finitely many conditions at any one stage, to perform a similar process for $\mathscr{P}_{X}$.

Given $p \in \mathscr{P}_{X}$, we would like to build a ${ }^{*}$-decreasing sequence $\left\langle p_{n} \mid n<\omega\right\rangle$ below $p$, whose lower bound can be extended to force $a$ into a countable ground model set. Essentially we will do the following: In building $p_{n+1}$, we will be at stage $n$ of a fusion process for $\mathscr{Q}_{x}$ on finitely many coordinates $x$ (say, for $x \in F$ ). We will consider finitely many possible extensions $\bar{p}$ of $p_{n}$ such that, for each $x \in F, \bar{p}^{R}(x)$ is forced to be one of the conditions we zeroextend in the fusion process on $\mathscr{Q}_{x}$; if it is possible to zero-extend the $\bar{p}^{R}(x)$ so that the resulting $\overline{\bar{p}} \leq \bar{p}$ decides the value of $a$, we do so (as part of the extension step), and $\overline{\bar{p}}$ becomes a step on the way to determining $p_{n+1}$. In this way we find countably many possible values for $a$, and $p_{\omega}$, the lower bound of $\left\langle p_{n} \mid n<\omega\right\rangle$, can be extended (by carrying out stage $\omega$ of the fusion process on every $\mathscr{Q}_{x}$ ) to a condition $q$ that forces this set to be exhaustive. 
The actual proof encounters technical obstacles of the following sort. We will construct $q$ so that whenever $p^{\prime} \leq q$ and $p^{\prime}$ decides the value of $a$, at some stage we try to determine the value of $a$ by extending some $\bar{p}$ such that for every $x \in F$,

$$
M_{X\lceil x} \vDash\left(p^{\prime}\right)^{R}(x) \leq_{0}(\bar{p})^{R}(x) .
$$

However, this fact can be seen in the generic extension; in the ground model, we cannot tell which choice of $(\bar{p})^{R}(x)$ will actually correspond to the value of $\left(p^{\prime}\right)^{R}(x)$ (at least, not while limiting ourselves to zero-extensions of $\left.(\bar{p})^{R}(x)\right)$, and so $p^{\prime}$ may not suitably extend the condition $\bar{p}$ we explicitly considered. Nonetheless, we are able to guarantee that the value $p^{\prime}$ forces for $a$ is one of the values we explicitly determined.

Definition. Let $F$ be a finite subset of $X, p$ a condition, $k<\omega$, and $\left\{r_{i}(x) \mid i<\right.$ $k, x \in F\}=\mathscr{R}$ a finite collection of terms such that for all $x$ and $i$,

$$
\Vdash_{x} r_{i}(x) \in\left(p^{Q}(x)\right)_{0} .
$$

(Recall that $\left(p^{Q}(x)\right)_{0}$, the first coordinate of a condition in $\mathscr{Q}_{x}$, is itself a set of conditions.) An assignment associated with $F$ and $\mathscr{R}$ is a map $I$ with domain $F$, such that for all $x \in F, I(x)$ is a term for forcing with $\mathscr{P}_{X\lceil x}$ and

$$
\Vdash_{x}(\exists i<k)\left[I(x)=r_{i}(x)\right] .
$$

For an assignment $I$ and a condition $q \leq p$, define a condition $q(I)$ by setting

$$
q(I)(x)= \begin{cases}\left\langle q^{Q}(x), I(x)\right\rangle, & x \in F, \\ q(x), & x \notin F .\end{cases}
$$

Define the notion of $(F, 0)$-extension by saying, for any conditions $p$ and $q$,

$$
\begin{aligned}
p \leq_{(F, 0)} q \text { iff } \quad & p \leq q \text { and for all } x \in F, \\
& p \nmid x \Vdash\left(p^{R}(x) \leq_{0} q^{R}(x)\right) .
\end{aligned}
$$

Now we can be more specific about the technical problems mentioned above. When building $p_{n+1}$, we are considering $F$ and $\mathscr{R}$. ( $\mathscr{R}$ is determined by the fusion process on $\mathscr{Q}_{x}$.) We want to successively consider all associated assignments $I,(F, 0)$-extend $p_{n}(I)$ to decide the value of $a$, and collect those values to form $A^{n}$.

The problem is that the quantifier $(\exists i)$ in the definition of assignment cannot be pulled down to the ground model, so that there are infinitely many such assignments. For no finite set $\mathscr{F}$ is it true that whenever there is some $I$ such that $q \leq_{(F, 0)} p_{n}(I)$, then for a further $(F, 0)$-extension $\bar{q}$ of $q$, there is $J \in \mathscr{I}$ such that $\bar{q} \leq_{(F, 0)} p_{n}(J)$. We can get away with considering only finitely many assignments because of the following lemma.

Lemma 10. Suppose $F$ has cardinality $n, \mathscr{R}=\left\{r_{i}(x) \mid i<k, x \in F\right\},\left\{I_{j} \mid j<\right.$ $m\}$ are assignments associated with $F$ and $\mathscr{R}, q$ is a condition, $a$ is a term 
for an element of the ground model, and $\left\{b_{j} \mid j<m\right\}$ are distinct elements of the ground model such that

$$
(\forall j<m)\left[q\left(I_{j}\right) \Vdash a=b_{j}\right] .
$$

Then $m \leq k^{n}$.

Proof. Induction on $n$. If $n=0$ and $m>1$, for any assignment $I$ we have $q(I)=q$, and so

$$
q \Vdash a=b_{0} \text { and } q \Vdash a=b_{i},
$$

a contradiction.

Suppose $\operatorname{CARD}(F)=n+1$ and $m>k^{n+1}$. Let $x$ be a minimal element of $F$. For each $h<m, I_{h}(x)$ is forced to be one of $\left\{r_{i}(x) \mid i>k\right\}$. Extend $q$ to $\overline{\bar{q}}$, by extending $q \nmid x$ without changing the rest of $q$, to decide for each $h<m$ the value of $i$ such that $I_{h}(x)=r_{i}(x)$. Then there are $i<k$ and $H$ of cardinality greater than $k^{n}$ such that for $h \in H$

$$
\overline{\bar{q}}\left\lceil x \Vdash I_{h}(x)=r_{i}(x)\right. \text {. }
$$

Let $\bar{F}=F-\{x\}, \bar{m}=\operatorname{CARD}(H), \bar{B}=\left\{b_{h} \mid h \in H\right\}, \bar{I}=\left\{I_{h}|\bar{F}| h \in H\right\}$, and for some $\bar{h} \in H$,

$$
\bar{q}=\overline{\bar{q}}\left(I_{\bar{h}}\right) .
$$

As $\overline{\bar{q}} \nmid x=\bar{q} \mid x$, and as $\bar{q} \uparrow x$ thus forces $\bar{q}(x)=r_{i}(x)$, whenever $h \in H$, $\bar{q}\left(I_{h} \backslash \bar{F}\right) \leq q\left(I_{h}\right)$; and so we have a contradiction to the inductive hypothesis.

Lemma 11. Let $X$ be any support partial order and $\mathscr{P}_{X}$ the mixed-support iteration of $\left\langle\mathscr{Q}_{x} * \mathscr{R}_{x} \mid x \in X\right\rangle$, where each $\mathscr{Q}_{x} * \mathscr{R}_{x}$ is forced to be the decomposition of a perfect tree forcing partial order. Let a be a term for an element of the ground model, and $p \in \mathscr{P}_{X}$. Then there are a countable ground model set $A$ and a condition $q \leq^{*} p$ such that $q \Vdash a \in A$.

Proof. Without loss of generality, we may assume $\operatorname{root}(p)=\varnothing$. The condition $q$ will extend the lower bound of a ${ }^{*}$-decreasing sequence $\left\langle p_{n} \mid n<\omega\right\rangle$, and $A$ will be the union of a sequence of finite sets $\left\langle A^{n} \mid n<\omega\right\rangle$.

Let the limit of $\left\langle p_{n} \mid n<\omega\right\rangle$ be denoted $p_{\omega}$.

We will determine, in the ground model, the sequence $\left\langle p_{n} \mid n<\omega\right\rangle$ simultaneously with a fusion process for $\mathscr{Q}_{x}$ for every $x \in \operatorname{supp}\left(p_{\omega}\right)$. Determining the fusion process will determine the sequence, by setting $p_{n}^{Q}(x)$ to be that condition $\left\langle p_{n}, \mathscr{A}_{n}\right\rangle$ in $\mathscr{Q}_{x}$ chosen at stage $n$ of the fusion process. Finally, we will let $q$ be determined by setting $q^{Q}(x)$ to be that condition $\langle Q, \mathscr{B}\rangle$ arrived at in stage $\omega$ of the fusion process.

To specify the construction, then, we must specify the arbitrary steps in the fusion process and the construction of the finite sets $A^{n}$.

Let $\langle F(n) \mid n<\omega\rangle$ decompose $\operatorname{supp}\left(p_{\omega}\right)$ as an increasing union of finite sets, $\operatorname{CARD}(F(n))=n$.

Before beginning stage 0 of the construction, set $p_{-1}=1$, and $p_{0}=p$. 
At the beginning of stage $n$ of the construction, we will have determined $\left\{A^{m} \mid m<n\right\}$, the condition $p_{n}$, and the first $n-1$ stages of a fusion process for $\mathscr{Q}_{x}$ for all $x \in \operatorname{supp}\left(p_{n-1}\right)$. For remaining $x \in \operatorname{supp}\left(p_{n}\right)$, let the first $n-1$ stages of the fusion process for $\mathscr{Q}_{x}$ be given by $\left\langle p_{0}, \mathscr{A}_{0}\right\rangle=\cdots=\left\langle p_{n}, \mathscr{A}_{n}\right\rangle=$ $p_{n}^{Q}(x)$, and the extension step is to do nothing.

Continue the fusion process for all $\mathscr{Q}_{x}, x \in \operatorname{supp}\left(p_{n}\right)$, up to the extension step.

For all $x$ which are not in $F(n)$, the extension step will be to do nothing.

For $x \in F(n)$, there are $n$ conditions (called $s_{m}(n), m<n$, in the definition of a fusion sequence for $\mathscr{Q}$ ) to be $n$-extended at the extension step. We can name them in the ground model by $\left\{s_{i}^{0}(x) \mid i<n\right\}$. Each has $2^{n}$ nodes $\sigma$ on its $n$th splitting level, denoted in the ground model by $\left\{\sigma_{i j}^{0}(x) \mid j<2^{n}\right\}$, and each $\left(s_{m}(n)\right)_{\sigma}$ can be altered be changing $\sigma \uparrow n$ to produce $2^{n}$ new conditions, denoted in the ground model by $\left\{r_{i j k}^{0}(x) \mid k<2^{n}\right\}$. We will inductively define $s_{n}^{m}, \sigma_{i j}^{m}, r_{i j k}^{m}$, so it is forced that $s_{i}^{m}(x) n$-extends $s_{i}^{0}(x)$, and $\sigma_{i j}^{m}(x), r_{i j k}^{m}(x)$ are related to $s_{i}^{m}(x)$ exactly as $\sigma_{i j}^{0}(x), r_{i j k}^{0}(x)$ are related to $s_{i}^{0}(x)$.

Define an $m$-assignment to be an assignment associated with $F(n)$ and

$$
\left\{r_{i j k}^{m}(x) \mid i, j, k \text { as above, } x \in F(n)\right\} \text {. }
$$

We will also inductively define a sequence of conditions $q_{m}$ and sets $B_{m}$.

Let $q_{0}=p_{n}$ and $B_{0}=\varnothing$.

Given all objects defined for $m$, suppose there are an $m$-assignment $I$, an element $b \notin B_{m}$, and a condition $q_{m+1} \leq_{(F(n), 0)} q_{m}(I)$, such that

$$
q_{m+1} \Vdash a=b .
$$

Then choose such a $q_{m+1}$, define $B_{m+1}=B_{m} \cup\{b\}$, and define new terms $s_{i}^{m+1}(x), \sigma_{i j}^{m+1}(x), r_{i j k}^{m+1}(x)$ for which it is forced:

There is a triple $\langle i j k\rangle$ with $I(x)=r_{i j k}^{m}(x)$. For this triple, if $\bar{r}$ is the restriction of $s_{i}^{m}(x)$ to $\sigma_{i j}^{m}(x)$ and $I(x)$ was obtained by replacing $\operatorname{stem}(\bar{r})$ with $\sigma$, and $r$ is obtained from $q_{m+1}^{R}(x)$ by replacing $\sigma$ with $\operatorname{stem}(\bar{r})$ (i.e., by changing the stem back again), then

$$
s_{i}^{m+1}(x)=\operatorname{Am}_{n}\left(r, s_{i}^{m}(x)\right),
$$

and for all other $i^{1}$,

$$
\left.s_{i^{1}}^{m+1}(x)=s_{i^{1}}^{m}(x)\right) .
$$

The $\sigma^{m+1}$ and $r^{m+1}$ are determined by the $s^{m+1}$.

We know by Lemma 10 that for some $m$, no such $q_{m+1}$ exists. For such $m$ define $A^{n+1}=B_{m}$; for $x \in F(n)$, the extension step of stage $n$ of the fusion process on $\mathscr{Q}_{x}$ is to replace each $s_{i}^{0}(x)$ by $s_{i}^{m}(x)$; for all $x \in \operatorname{supp}\left(p_{n}\right)$, the final step of stage $n$ of the fusion process on $\mathscr{Q}_{x}$ is to set $\left\langle p_{n+1}, \mathscr{A}_{n+1}\right\rangle=q_{m}^{Q}(x)$. 
To complete the $n$th stage of the main inductive construction, set for all $x \in X$,

$$
p_{n+1}^{Q}(x)=q_{m}^{Q}(x) \text {. }
$$

Now we have defined $\left\langle p_{n}, A^{n} \mid n<\omega\right\rangle$. Let $p_{\omega}$ be the limit of the $p_{n}$,

$$
A=\bigcup\left\{A^{n} \mid n<\delta\right\},
$$

and $q \leq^{*} p_{\omega}$ be defined by

$$
q^{Q}(x)=\left\{\begin{array}{l}
\langle Q, \mathscr{B}\rangle \text { as produced in the fusion for } \mathscr{Q}_{x}, \quad x \in \operatorname{supp}\left(p_{\omega}\right), \\
1, \quad x \notin \operatorname{supp}\left(p_{\omega}\right) .
\end{array}\right.
$$

We must show that $q \Vdash a \in A$.

Suppose, then, that $\bar{q} \leq q$ and for some $b \in M, \bar{g} \Vdash a=b$. By construction, we can extend $\bar{q}$ so that for every $x$ in $\operatorname{root}(\bar{q}) \cap \operatorname{supp}\left(p_{\omega}\right)$, it is forced that for some stage $n$ of the inductive construction of $\left\langle p_{n} \mid n<\omega\right\rangle$

$$
\left(\exists\langle i, j, k\rangle\left[\bar{q}^{R}(x) \leq_{0} r_{i, j, k}^{m}(x)\right],\right.
$$

where $m$ is the greatest integer such that $q_{m}$ was defined at stage $n$.

We can assume that $\bar{q}$ decides the choice of $n$ for every $x \in \operatorname{root}(\bar{q}) \cap \operatorname{supp}\left(p_{\omega}\right)$. (Suppose not. Let $\alpha(\bar{q})$ be the largest ordinal $\alpha$ such that, for some such $x$ of height $\alpha$ in $X$, the associated $n, n(x)$, has not been decided by $\bar{q}$. As $\operatorname{root}(\bar{q})$ is finite, we can change $\bar{q}$ to $\overline{\bar{q}}$ by strengthening $\bar{q} \uparrow\{y \mid \operatorname{ht}(y)<\alpha\}$, without changing the rest of $\bar{q}$, so that $\bar{q}$ decides $n(x)$ for any $x$ in $\operatorname{root}(\bar{q}) \cap \operatorname{supp}\left(p_{\omega}\right)$ of height $\alpha$. Since $\operatorname{root}(\bar{q})$ has been affected only below $\alpha, \alpha(\overline{\bar{q}})<\alpha(\bar{q})$; by the well-foundedness of $X$, repeating this process finitely many times will produce a condition which does decide $n(x)$ for every relevant $x$.) Finally, by replacing each $\bar{q}^{R}(x)$ by a term for a suitable restriction, we can assume that the same $n$ works for every $x$.

Suppose the construction at stage $n$ halted with the definition of $q_{m}$ and $B_{m}$. As $\operatorname{root}(\bar{q}) \cap \operatorname{supp}\left(p_{\omega}\right) \subseteq F(n)$, there is an $m$-assignment $I$ such that, for $x \in \operatorname{root}(\bar{q}) \cap \operatorname{supp}\left(p_{\omega}\right)$, it is forced that $I(x)=(\bar{q})^{R}(x)$. The conditions $q_{m}(I)$ and $\bar{q}$ have a common extension $\bar{p}$, defined by

$$
\begin{aligned}
& (\bar{p})^{Q}(x)=(\bar{q})^{Q}(x), \\
& (\bar{p})^{R}(x)= \begin{cases}(\bar{q})^{R}(x), & x \in \operatorname{root}(\bar{q}), \\
\left(q_{m}(I)\right)^{R}(x), & x \notin \operatorname{root}(\bar{q}) .\end{cases}
\end{aligned}
$$

Now $\bar{p} \leq_{(F(n), 0)} q_{m}(I)$, and $\bar{p} \Vdash a=b$. Since no $q_{m+1}$ was defined, we can conclude that $b \in B_{m}$. Therefore, as desired, $b \in A$.

This proves the lemma.

As corollaries to this lemma, we have the following facts. 
Theorem 13. The mixed-support generalized iteration of perfect tree forcing decompositions preserves $\omega_{1}$.

Theorem 14. If $\mathscr{P}_{X}$ is the mixed-support iteration of perfect tree forcing decompositions and $\mathrm{CH}$ holds in the ground model, then

If $\operatorname{CARD}(X) \leq \omega_{1}$, then $\mathscr{P}_{X}$ has $a^{*}$-dense subset of size $\omega_{1}$, and $\mathscr{P}_{X}$ forces CH to hold.

If $X$ is $\omega_{2}$-like, then $\mathscr{P}_{X}$ has the $\omega_{2}$-chain condition, and preserves all cardinals.

\section{A FORCING PRINCIPLE}

In this section, we give an example of a forcing construction using a generalized iteration to produce a model in which the continuum is large. We show it is possible to obtain an arbitrarily large continuum together with the following forcing axiom:

If $\mathscr{P}$ is any forcing partial order with the perfect tree property, and $\mathscr{D}$ a collection of $\omega_{1}$ dense subsets of $\mathscr{P}$, then there is a $\mathscr{D}$-generic filter $G \subseteq \mathscr{P}$. The construction, of course, will be to force with a mixed-support iteration $\mathscr{P}_{X}$ of perfect tree forcing decompositions. We have just shown that if $X$ is $\omega_{2}$-like, this will preserve cardinals. By making $X$ large, we can make the continuum large. We will further exploit the fact that $X$ need not be well ordered to guarantee that for any suitable $\mathscr{D}$ and $\mathscr{P}$, at some intermediate stage of the iteration we added a $\mathscr{D}$-generic filter on $\mathscr{P}$.

\section{Theorem 15.}

$\operatorname{Con}(\mathrm{ZF}) \Rightarrow \operatorname{Con}\left(\mathrm{ZFC}+2^{\omega}\right.$ is arbitrarily large $+\mathrm{FP}\left(\right.$ perfect tree forcing, $\left.\left.\omega_{2}\right)\right)$.

The forcing principle $\operatorname{FP}(\mathscr{C}, \lambda)$ states that if $\mathscr{P}$ is a forcing partial order in the class $\mathscr{C}$, and $\mathscr{D}$ a collection of fewer than $\lambda$ dense subsets of $\mathscr{P}$, then there is a $\mathscr{D}$-generic filter on $\mathscr{P}$.

Definition. Let $X$ be any support partial order and $Y \subseteq Y$ be downwardclosed. An element $y \in X$ is an exact upper bound for $Y$ iff $X \mid y=Y$.

The idea of the proof is to iterate along an $\omega_{2}$-like support partial order $X$ of size $\kappa$, where $\kappa$ is a regular cardinal greater than $\omega_{1}$, at each coordinate $x$ forcing with a perfect tree forcing decomposition $\mathscr{Q}_{x} * \mathscr{R}_{x}$ in $M_{X \mid x}$. This will preserve cardinals and force $2^{\omega}=\kappa$.

If, in the generic extension $M[G], \mathscr{P}$ is a perfect tree forcing partial order and $\mathscr{D}$ a size $\omega_{1}$ collection of dense subsets of $\mathscr{P}$, then this situation can be reflected to an initial segment of the iteration $M_{Y}$, where $Y$ has size $\omega_{1}$. Then we will add a $\mathscr{D}$-generic subset of $\mathscr{P}$ by guaranteeing that, for some exact upper bound $y$ for $Y, \mathscr{Q}_{y} * \mathscr{P}_{y}$ is the decomposition of the version of $\mathscr{P}$ reflected in $M_{Y}$.

Two advantages of generalized iteration over standard iteration appear in this outline. First, we can choose an $\omega_{2}$-like support partial order of size $\kappa$, making 
the continuum large. Second, having reflected a situation regarding $\mathscr{P} \in M[G]$ to $\overline{\mathscr{P}} \in M_{Y}$, we need not worry that over $M_{Y}$ we forced, not with $\mathscr{\mathscr { P }}$, but with some other forcing partial order instead; if $Y$ has enough exact upper bounds in $X$ we can force with every perfect tree forcing decomposition in $M_{Y}$. This guarantees that every $\mathscr{P}$ and $\mathscr{D}$ will be taken care of without appealing to a large cardinal reflection argument.

Proof. Let $M \vDash \mathrm{ZFC}+\mathrm{GCH}$, and $\kappa \geq \omega_{2}$ be a regular cardinal in $M$.

In $M$, let $X$ be an $\omega_{2}$-like support partial order of cardinality $\kappa$, such that every downward-closed $Y \subseteq X$ of size at most $\omega_{1}$ has at least $\omega_{2}$ exact upper bounds in $X$.

Let $\mathscr{P}_{X}$ be a mixed-support iteration of perfect tree forcing decompositions $\left\langle\mathscr{Q}_{x} * \mathscr{R}_{x} \mid x \in X\right\rangle$, such that if $Y \subseteq X$ is downward-closed and of size at most $\omega_{1}$, every perfect tree forcing decomposition in $M_{Y}$ will be $\mathscr{Q}_{x} * \mathscr{R}_{x}$ for some exact upper bound $x$ for $Y$. (Because $\mathscr{P}_{Y}$ preserves cardinals and $\mathrm{GCH}$, and because from the definition of the perfect tree property it follows that under $\mathrm{GCH}$ there are only $\omega_{2}$ many forcing partial orders with this property, $Y$ has enough exact upper bounds to allow us to do this.)

We know $\mathscr{P}_{X}$ preserves cardinals.

If $G$ is $\mathscr{P}_{X}$-generic over $M$, then in $M[G], 2^{\omega}=\kappa$.

It remains to see that

$$
M[G] \vDash \mathrm{FP}\left(\text { perfect tree forcing, } \omega_{2}\right) .
$$

Suppose $\mathscr{P}$ has the perfect tree property in $M[G]$, and $\left\langle D_{\alpha} \mid \alpha<\omega_{1}\right\rangle$ is a collection of dense subsets of $\mathscr{P}$. We will consider the universe of $\mathscr{P}$ to be a subset of some cardinal $\delta$.

Because $\mathscr{P}_{X}$ has the $\omega_{2}$ chain condition, if $S$ is a term for a size $\omega_{1}$ structure in $M[G]$ whose universe is a set of ordinals, then there is a size $\omega_{1} Y \subseteq X$ in $M$ for which $\mathscr{P}_{Y}$ completely determines $S$. In particular, it is forced that $S \in M_{Y}$. (For each $n$-ary relation $R$ of the structure $S$, and each $\alpha_{1}, \ldots, \alpha_{n}<\omega_{1}$, there is a maximal antichain $C\left(R, \alpha_{1}, \ldots, \alpha_{n}\right)$ of conditions determining the $\left(\alpha_{1}\right)$ th $, \ldots,\left(\alpha_{n}\right)$ th elements of $S$ and deciding whether $R$ holds on these elements. Let

$$
\begin{array}{r}
Y=\left\{y \in X \mid(\exists R)\left(\exists\left\langle\alpha_{1}, \ldots, \alpha_{n}\right\rangle\right)\left(\exists p \in C\left(R, \alpha_{1}, \ldots, \alpha_{n}\right)\right)\right. \\
(\exists x \in \operatorname{supp}(p))[y<x]\} .
\end{array}
$$

Since there are $\omega_{1}$ antichains of the form $C\left(R, \alpha_{1}, \ldots, \alpha_{n}\right)$, each having size $\omega_{1}$, each condition $p$ having countable support, and each $x$ having at most countably many predecessors, $Y$ has size $\omega_{1}$. (Maximal antichains of $\mathscr{P}_{X}$ completely determining the structure $S$ lie in $\mathscr{P}_{Y}$.)

If $Y \subseteq X$ is such that any countable subset of $Y$ has an upper bound in $Y$, then every countable sequence of ordinals in $M_{Y}$ is actually in $M_{Y \nmid y}$ for some $y \in Y$. (Let $\left\langle\alpha_{n} \mid n<\omega\right\rangle$ be a term for a countable sequence of ordinals in $M_{Y}$. In $M_{Y}$, for each $n$ there is $p_{n} \in G_{Y}$ deciding the value of $\alpha_{n}$, and 
$S=\bigcup\left\{\operatorname{supp}\left(p_{n}\right) \mid n<\omega\right\}$ is a countable subset of $Y$. Lemma 11 of $\S 2$ can be used to show that $S$ is contained in a countable set $T$ in $M$. But then if $y$ is an upper bound for $T$ in $Y,\left\langle\alpha_{n} \mid n<\omega\right\rangle \in M_{Y \mid y}$.)

In $M$, choose inductively for $\alpha \leq \omega_{1}, Y(\alpha) \subseteq X$ and $P(\alpha) \subseteq \delta$ (recall that $\delta$ is the universe of the forcing partial order $\mathscr{P} \in M[G])$ such that:

(i) $Y(\alpha)$ and $P(\alpha)$ have size $\omega_{1}$.

(ii) For $\alpha<\omega_{1}, Y(\alpha) \subseteq Y(\alpha+1)$ and $P(\alpha) \subseteq P(\alpha+1)$. If $\alpha$ is a limit ordinal, $P(\alpha)=\bigcup\{P(\beta) \mid \beta<\alpha\}$ and $Y(\alpha)=\bigcup\{Y(\beta) \mid \beta<\alpha\}$.

(iii) The structure of $\mathscr{P}$ (ie, $\leq, \leq_{0}$, splitting levels, restrictions, amalgamations, changes of stem, finite unions) restricted to $P(\alpha)$ is decided by $\mathscr{P}_{Y(\alpha+1)}$. Each $D_{\gamma} \cap P(\alpha)$ is decided by $\mathscr{P}_{Y(\alpha+1)}$.

(iv) Every countable subset of $Y(\alpha)$ has an upper bound in $Y(\alpha+1)$.

(v) For each $\gamma<\omega_{1}$ and $p \in P(\alpha)$, it is forced that there is a $q \in P(\alpha+1)$ such that $q \leq p$ and $q \in D_{\gamma}$. (There is a size $\omega_{1}$ maximal antichain deciding the least such $q$. Simply add all $\omega_{1}$ many possibilities to $p(\alpha+1)$.)

(vi) It is forced that any fusion sequence from $P(\alpha)$ in $M_{Y(\alpha)}$ has a fusion in $P(\alpha+1)$. (Similarly, there are only $\omega_{1}$ fusion sequences from $P(\alpha)$ in $M_{Y(\alpha)}$, and for each $\beta<\omega_{1}$, only $\omega_{1}$ possibilities for the fusion of the $\beta$ th sequence.)

Let $Y=Y\left(\omega_{1}\right)$ and $P\left(\omega_{1}\right)$. Then:

(i) $Y$ and $P$ have size $\omega_{1}$, and every countable subset of $Y$ has an upper bound in $Y$. (Hence, every fusion sequence from $P$ in $M_{Y}$ is actually in $M_{Y \mid y}$ for some $y \in Y$, and thus in $M_{Y(\alpha)}$ for some $\alpha<\omega$.)

(ii) It is forced that the structure of $\mathscr{P}$ restricted to $P$ is in $M_{Y}$ and that any fusion sequence from $P$ in $M_{Y}$ has a fusion in $P$.

(iii) Each $D_{\gamma} \cap P$ is forced to be dense in $P$ and to be an element of $M_{Y}$.

Then $P$, as a suborder of $\mathscr{P}$, is a perfect tree forcing partial order in $M_{Y}$. For some exact upper bound $y$ for $Y, \mathscr{Q}_{y} * \mathscr{R}_{y}$ is the decomposition of $P$ in $M_{Y}$; thus, $G(y)$ adds a $P$-generic over $M_{Y}$. In $M[G], G(y)$ adds a $\left\langle D_{\gamma}\right| \gamma<$ $\left.\omega_{1}\right\rangle$-generic filter for $\mathscr{P}$.

This proves $\mathrm{FP}$ (perfect tree forcing, $\left.\omega_{2}\right)$ in $M[G]$.

\section{Conclusion}

This paper has essentially addressed one technical question about generalized iteration, that of finding a class of forcing partial orders that can be iterated while preserving $\omega_{1}$, and given one application, the consistency of a forcing principle with large continuum. Open questions include most of the technical questions that have been dealt with in the case of standard iterations. It is to be hoped that there will be more applications of this technique to producing models with large continuum.

One specific question, with its associated technical challenge, is the following. Standard iteration produces a model of MA + large continuum. Generalized iteration produces a model of $\mathrm{FP}\left(\right.$ perfect tree forcing, $\left.\omega_{2}\right)+$ large 
continuum. Neither construction works in the other context. Is it possible to produce, from a model of $\mathrm{ZFC}$, a model of $\mathrm{MA}_{\omega_{1}}+\mathrm{FP}$ (perfect tree forcing, $\left.\omega_{2}\right)+$ large continuum? This is a step toward answering the question: What forcing principles are consistent with large continuum? The strongest possible forcing principle for meeting $\omega_{1}$ dense sets is $\operatorname{FP}\left(\mathscr{C}, \omega_{2}\right)$ where $\mathscr{C}$ is the class of forcing partial orders that preserve stationary subsets of $\omega_{1}$; this axiom was christened Martin's Maximum Principle by Foreman, Magidor, and Shelah, who proved it consistent assuming the consistency of a supercompact cardinal. They give an upper bound for an answer to this last question, by showing that Martin's Maximum Principle implies the continuum is $\omega_{2}$. Todorcevic has shown that $\mathrm{PFA}^{+}$, a technical strengthening of the proper forcing axiom (FP(proper forcing, $\left.\omega_{2}\right)$ ), likewise implies that the continuum is $\omega_{2}$. Whether the proper forcing axiom can be consistent with the continuum greater than $\omega_{2}$ is still unknown.

\section{APPENDIX}

Here we give some examples of standard forcing partial orders to add reals which are perfect tree forcings and show how they can be put into this framework.

Example. Sacks forcing [17] is defined by

$$
\mathscr{P}=\mathscr{T} ; \quad s \leq t \quad \text { iff } s \subseteq t .
$$

This is a perfect tree forcing under the definitions:

$$
s \leq_{0} t \quad \text { iff } \quad s \leq t
$$

if $\sigma \in S_{n}(t), s \leq_{0}(t)_{\sigma}$, then for all $\tau \in S_{n}(t)$,

$$
\left(\operatorname{Am}_{n}(s, t)\right)_{\tau}= \begin{cases}s, & \tau=\sigma, \\ (t)_{\tau}, & \tau \neq \sigma .\end{cases}
$$

Example. Prikry-Silver forcing [6] is defined by

$$
\begin{gathered}
\mathscr{P}=\{t \in \mathscr{T} \mid \text { if } \delta, \tau \in t,|\delta|=|\tau|, \text { then } \delta p \in t \Rightarrow \tau(p \in t\}, \\
s \leq t \quad \text { iff } s \subseteq t .
\end{gathered}
$$

This is a perfect tree forcing under the definitions:

$$
s \leq_{0} t \quad \text { iff } \quad s \leq t
$$

if $\sigma \in S_{n}(t), s \leq_{0}(t)_{\sigma}$, then for all $\tau \in S_{n}(t)$,

$$
\left(\operatorname{Am}_{n}(s, t)\right)_{\tau}=\left\{\tau^{\frown} p \mid \sigma^{\frown} p \in x\right\} \cup\{p \mid p \subset \tau\} .
$$

Example . Laver forcing [13] is defined by

$$
\begin{gathered}
\mathscr{Q}=\left\{t \subseteq{ }^{\omega} \omega \mid t \text { is downward-closed and for all } \sigma \in t,\right. \\
\text { if stem } \left.(t) \subseteq \sigma \text {, then }\left\{n \mid \sigma^{-} n \in t\right\} \text { is infinite }\right\}, \\
s \leq t \text { iff } s \subseteq t .
\end{gathered}
$$


The map $f:{ }^{\omega} \omega \rightarrow{ }^{\omega} 2$ defined by

$$
\begin{gathered}
f(\varnothing)=\varnothing, \\
f\left(\sigma^{\frown} n\right)=f(\sigma)^{\frown}(\text { a sequence of } n \text { 0's })^{\frown} 1,
\end{gathered}
$$

induces an order-preserving map $\bar{f}: \mathscr{Q} \rightarrow \mathscr{T}$ :

$$
\bar{f}(t)=f^{\prime \prime} t .
$$

The image of $\mathscr{Q}$ under $\bar{f}$ is

$$
\mathscr{P}=\left\{t \in \mathscr{T} \mid \text { if } \operatorname{stem}(t) \subseteq \sigma \text { and } \sigma \in t \text { then } \sigma^{\frown} 0 \in t\right\},
$$

which is a perfect tree forcing under the definitions:

$$
s \leq_{0} t \text { if } s \leq t \text { and stem }(s)=\operatorname{stem}(t) \frown \text { (a sequence of } 0 \text { 's); }
$$

if $\sigma \in S_{n}(t), s \leq_{0}(t)_{\sigma}$, then for all $\tau \in S_{n}(t)$,

$$
\left(\operatorname{Am}_{n}(s, t)\right)_{\tau}= \begin{cases}s, & \tau=\sigma \\ (t)_{t}, & \tau \neq \sigma .\end{cases}
$$

Example. Mathias forcing [15] is defined by $\mathscr{Q}=\{\langle\sigma, X\rangle \mid$ for some $n \in \omega, \sigma \in$ $\eta$ and $X$ is an infinite subset of $\omega-n\},\langle\sigma, X\rangle \leq\langle\tau, Y\rangle$ iff $\tau \subseteq \sigma, X \subseteq Y$, and for all $n \in$ length $(\sigma)$ - length $(\tau), \sigma(n)=1 \Rightarrow n \in X$.

An order-preserving map from $\mathscr{Q}$ to $\mathscr{T}$ is given by

$$
\begin{aligned}
& f(\langle\sigma, X\rangle \vDash\{\tau \mid \tau \subseteq \sigma \text { or } \sigma \subseteq \tau, \text { and for all } \\
& \qquad n \in \text { length }(\tau)-\text { length }(\sigma), \tau(n)=1 \Rightarrow n \in X\} .
\end{aligned}
$$

The image of $\mathscr{Q}$ under $f$ is

$\mathscr{P}=\left\{t \mid t\right.$ is a condition for Laver forcing, and if $\sigma \in{ }^{n} 2 \cap t$

$$
\text { and } \left.\tau \in{ }^{n} 2 \cap t \text {, then for all } p, \sigma^{-} p \in t \text { iff } \tau^{\uparrow} p \in t\right\} \text {, }
$$

which is a perfect tree forcing if we define $\leq_{0}$ as for Laver forcing, and amalgamation by, given $\sigma \in S_{n}(t)$ and $s \leq_{0}(t)_{\sigma}$, then for all $\tau \in S_{n}(t)$,

$$
\begin{gathered}
\left(\operatorname{Am}_{n}(s, t)\right)_{\tau} \text { is obtained from } s \text { by a change } \\
\text { of stem that replaces } \sigma \text { with } \tau \text {. }
\end{gathered}
$$

The definition of perfect tree forcing given here was chosen for concreteness and relative simplicity, to facilitate the definition of the decomposition $\mathscr{Q} * \mathscr{R}$ and the proof that $\mathscr{Q} * \mathscr{R}$ actually adds a $\mathscr{P}$-generic. In fact, the definition is much narrower than the proof of the iteration theorem requires. That proof uses the following facts about $\mathscr{Q} * \mathscr{R}$ :

(i) $\mathscr{Q}$ is a countably closed forcing partial order, which generically adds a forcing partial order $\mathscr{R}$ with ordering $\leq$ and a refinement of the ordering $\leq_{0}$.

(ii) There is an $(\omega+1)$-stage fusion process for $\mathscr{Q}$ such as the one we have sketched. Stage $n$ allows finitely many potential conditions in $\mathscr{R}$, $\{r(i, n) \mid i<f(n)\}$, to be successively zero-extended, arbitrarily, to obtain 
$\left.\left\{r^{\prime}(i, n)\right\} \mid i<f(n)\right\}$, and allows the condition $q_{n}$ in $\mathscr{Q}$ to be arbitrarily extended to $q_{n+1}$. Stage $\omega$ produces a condition $q$ extending all of the $q_{n}$, such that $q$ forces:

$$
(\forall r \in \mathscr{R})(\exists m)(\forall n>m)(\exists s \leq r)(\exists i<f(n))\left[s \leq_{0} r^{\prime}(i, n)\right] .
$$

(iii) The function $f$ is eventually bounded by a recursive function. Some such requirement is necessary because the proof of the iteration theorem required us to predict in the ground model exactly how many conditions are to be zero-extended at stage $n$ of the fusion process on $\mathscr{Q}_{x}$.

The following quickly sketched examples fit into this rather loose characterization and illustrate how the scope of the iteration theorem, and of the theorem in $\S 4$, can be expanded. We give, without proofs, enough details to indicate that the proof of the iteration theorem applies to each.

Example. Solovay's forcing notion to almost-disjoint code a set $B \subseteq \omega_{1}$ [10] is defined from a collection of almost disjoint subsets of $\omega,\left\{b_{\alpha} \mid \alpha<\omega_{1}\right\}$, by:

$$
\begin{gathered}
\overline{\mathscr{Q}}=\left\{\langle\sigma, S\rangle \mid \sigma \in \stackrel{\omega}{\omega}^{2}, S \in\left[\omega_{1}\right]^{<\omega}\right\}, \\
\langle\sigma, S\rangle \leq\langle\tau, T\rangle \text { iff } \tau \subseteq \sigma, T \subseteq S, \text { and for all } i \in \text { length }(\sigma)-\text { length }(\tau), \\
\sigma(i) \notin \bigcup\left\{b_{\alpha} \mid \alpha \in T\right\} .
\end{gathered}
$$

We define a supplementary partial order, $\mathscr{P}$ :

$$
\begin{gathered}
\mathscr{P}=\left\{\langle t, f\rangle \mid t=\left({ }^{\omega} 2\right)_{\sigma} \text { for some } \sigma, \text { and } f: t \rightarrow\left[\omega_{1}\right]^{<\omega}\right. \\
\text { is such that if } p \subseteq \tau \text { then } f(p) \subseteq f(\tau)\}, \\
\langle t, f\rangle \leq\langle s, g\rangle \text { iff } t \subseteq s, \quad \text { for all } \sigma \in t, g(\sigma) \subseteq f(\sigma),
\end{gathered}
$$

and

$$
\langle\operatorname{stem}(t), f(\operatorname{stem}(t))\rangle \text { extends }\langle\operatorname{stem}(s), g(\operatorname{stem}(s))\rangle \text { in } \overline{\mathscr{Q}} \text {. }
$$

If $\mathscr{G}$ is $\mathscr{P}$-generic, then

$$
\{\langle\sigma, S\rangle \mid \text { for some }\langle t, f\rangle \in \mathscr{G}, \sigma=\operatorname{stem}(t) \text { and } f(\sigma)=S\}
$$

is $\overline{\mathscr{Q}}$-generic. An Axiom A structure with finite splitting can be put on $\mathscr{P}$ :

$$
\begin{aligned}
& \langle t, f\rangle \leq_{0}\langle s, g\rangle \quad \text { iff }\langle t, f\rangle \leq\langle s, g\rangle \text { and } t=s, \\
& \langle t, f\rangle \leq_{n}\langle s, g\rangle \quad \text { iff }\langle t, f\rangle \leq_{0}\langle s, g\rangle \text { and } \\
& \left(\forall m\langle n)\left(\forall \sigma \in^{m} 2\right)\left[f\left(\operatorname{stem}(t)^{-} \sigma\right)=g\left(\operatorname{stem}(s)^{-} \sigma\right)\right],\right.
\end{aligned}
$$

if $\operatorname{stem}(t) \subseteq \sigma$

$$
(\langle t, f\rangle)_{\sigma}=\left\langle(t)_{\sigma}, f(t)_{\sigma}\right\rangle,
$$

if $\langle t, f\rangle \leq_{0}\langle s, g\rangle_{\sigma}$, where for some $\tau \in{ }^{n} 2, \sigma=\operatorname{stem}(s)^{\complement} \tau$

$$
\operatorname{Am}_{n}(\langle t, f\rangle,\langle s, g\rangle)=\langle s, h\rangle
$$

where

$$
h(t)= \begin{cases}f(\tau), & \sigma \subseteq \tau, \\ g(t), & \text { otherwise }\end{cases}
$$


A $\mathscr{P}$-generic can be added by the decomposition $\mathscr{Q} * \mathscr{R}: \mathscr{Q}=\{\langle P, \mathscr{A}\rangle \mid P$ is a countable subset of $\mathscr{P}, \mathscr{A}$ is a countable collection of subsets of $\mathscr{P}$, for every $A \in \mathscr{A}$ and $\langle t, f\rangle \in P$ there is $\langle s, g\rangle \in P$ such that $\langle s, g\rangle \leq_{0}\langle t, f\rangle$ and $A$ is predense in $\mathscr{P}$ below $\langle s, g\rangle\}$,

$$
\langle P, \mathscr{A}\rangle \leq\langle Q, \mathscr{B}\rangle \quad \text { iff } \quad Q \subseteq P \text { and } \mathscr{B} \subseteq \mathscr{A} .
$$

If $\mathscr{G} \subseteq \mathscr{Q}$ is generic, then

$$
\mathscr{R}=\bigcup\{P \mid\langle P, \varnothing\rangle \in \mathscr{G}\},
$$

ordered as a suborder of $\mathscr{P}$.

Here, $\mathscr{Q}$ has the usual fusion process, except that the complication of changing stems is avoided.

Example. Shelah's forcing partial order to add a generic Sacks condition is a modification of Sacks forcing [15] defined by:

$$
\begin{aligned}
& \mathscr{P} \text { denotes Sacks forcing, } \\
& \overline{\mathscr{Q}}=\{\langle t, u\rangle \mid t \in \mathscr{P}, u \text { is a finite initial segment of } t\}, \\
& \langle t, u\rangle \leq\langle s, v\rangle \text { iff } t \subseteq s \text { and } v \subseteq u .
\end{aligned}
$$

An Axiom A structure with finite splitting can be put on $\overline{\mathscr{Q}}$ :

$$
\begin{aligned}
& \langle t, u\rangle \leq_{0}\langle s, v\rangle \quad \text { iff } t \subseteq s \text { and } u=v, \\
& \langle t, u\rangle \leq_{n}\langle s, v\rangle \quad \text { iff } \quad u=v \text { and } t \leq_{n} s \text { as conditions in } \mathscr{P} ;
\end{aligned}
$$

if $u$ is bounded below $S_{n}(t), \Sigma \subseteq S_{n}(t)$, and for every $\tau \in u$ there is $\sigma \in \Sigma$ such that $\tau \leq \sigma$, then

$$
(\langle t, u\rangle)_{\Sigma}=\left\langle\bigcup\left\{(t)_{\sigma} \mid \sigma \in \Sigma\right\},\{\tau \mid(\exists \sigma \in \Sigma)[\tau \subset \sigma]\}\right\rangle ;
$$

if $\Sigma \subseteq S_{n}(t)$ and $\langle s, v\rangle \leq_{0}(\langle t, u\rangle)_{\sigma}$, then

$$
\operatorname{Am}_{n}(\langle s, v\rangle,\langle t, u\rangle)=\left\langle s \cup \bigcup\left\{(t)_{\tau} \mid \tau \in S_{n}(t)-\Sigma\right\}, u\right\rangle .
$$

A $\overline{\mathscr{Q}}$-generic can be added by the decomposition $\mathscr{Q} * \mathscr{R}: \mathscr{Q}=\{\langle P, \mathscr{A}\rangle \mid P$ is a countable subset of $\mathscr{P}, \mathscr{A}$ is a countable collection of subsets of $\bar{P}=$ $\{\langle t, u\rangle \in \overline{\mathscr{Q}} \mid t \in P\}$, for every $A \in \mathscr{A}$ and $\langle t, u\rangle \in \bar{P}$ there is $\langle s, v\rangle \in \bar{P}$ such that $\langle s, v\rangle \leq_{0}\langle t, u\rangle$ and $A$ is predense in $\overline{\mathscr{Q}}$ below $\left.\langle s, v\rangle\right\}$,

$$
\langle P, \mathscr{A}\rangle \leq\langle Q, \mathscr{B}\rangle \quad \text { iff } \quad Q \subseteq P \text { and } \mathscr{B} \subseteq \mathscr{A} \text {. }
$$

Here, the fusion process on $\mathscr{Q}$ actually builds fusion sequences from $\mathscr{P}$ (rather than from $\overline{\mathscr{Q}}$ ). At stage $n$, finitely many conditions $t \in P$ are $n$-extended by zero extending each $(\langle t, \varnothing\rangle)_{\Sigma}$, for $\Sigma \subset S_{n}(t)$, as a condition in $\overline{\mathscr{Q}}$.

Example. Countably closed forcing has a natural (trivial) Axiom A structure with no splitting.

Added in proof. Todorcevic has shown PFA itself implies the continuum is $\omega_{2}$. $\mathrm{He}$ and Veličkovic̀ have shown PFA can be weakened to $\mathrm{FP}$ (countable closed $\left.* \operatorname{ccc}, \omega_{2}\right)$. 


\section{REFERENCES}

1. J. Baumgartner, Iterated forcing, Surveys in Set Theory (A.R.D. Mathias, ed.), London Math. Soc. Lecture Note Ser. 87, Cambridge University Press, Cambridge, 1983, pp. 1-59.

2. J. Baumgartner and R. Laver, Iterated perfect set forcing, Ann. Math. Logic 17 (1979), 271-288.

3. P. Cohen, Set theory and the continuum hypothesis, Benjamin, New York, 1966.

4. K. Devlin, The Yorkshireman's guide to proper forcing, Surveys in Set Theory (A.R.D. Mathias, ed.), London Math. Soc. Lecture Note Ser. 87, Cambridge University Press, Cambridge, 1983, pp. 60-115.

5. E. Easton, Powers of regular cardinals, Ann. Math. Logic 1 (1970), 139-178.

6. S. Grigorieff, Combinatorics on ideals and forcing, Ann. Math. Logic 3 (1971), 363-394.

7. M. Groszek, $\omega_{1}^{*}$ as an initial segment of the degrees of constructibility.

8. M. Groszek and R. Laver, Finite groups of OD-conjugates.

9. T. Jech, Set theory, Academic Press, New York, 1978.

10. R. Jensen, Definable sets of minimal degree, Math. Logic and Foundations of Set Theory (Y. Bar-Hillel, ed.), North-Holland, Amsterdam, 1970, pp. 122-218.

11. R. Jensen and R. Solovay, Some applications of almost disjoint sets, Math. Logic and Foundations of Set Theory (Y. Bar-Hillel, ed.), North-Holland, Amsterdam, 1970, pp. 84-104.

12. K. Kunen, Set theory, Stud. Logic Foundations Math., vol. 102, North-Holland, Amsterdam, 1980.

13. R. Laver, On the consistency of Borel's conjecture, Acta Math. 137 (1976), 151-169.

14. D. Martin and R. Solovay, Internal Cohen extensions, Ann. Math. Logic 2 (1970), 143-178.

15. A. Mathias, Happy families, Ann. Math. Logic 12 (1977), 59-111.

16. A. Miller, Rational perfect set forcing.

17. G. Sacks, Forcing with perfect closed sets, Axiomatic Set Theory (D. Scott, ed.), Proc. Sympos. Pure Math., vol. 13, part 1, Amer. Math. Soc., Providence, R.I., 1971, pp. 331355.

18. S. Shelah, Proper forcing, Lecture Notes in Math., vol. 940, Springer-Verlag, Berlin, 1982.

19. R. Solovay and S. Tennenbaum, Iterated Cohen extensions and Souslin's problem, Ann. of Math. (2) 94 (1971), 201-245.

20. W. H. Woodin, The consistency of Borel's conjecture with large continuum.

Department of Mathematics, Dartmouth College, Hanover, New Hampshire 03755

Department of Mathematics, Pennsylvania State University, University Park, PennSYLVANIA 16802 